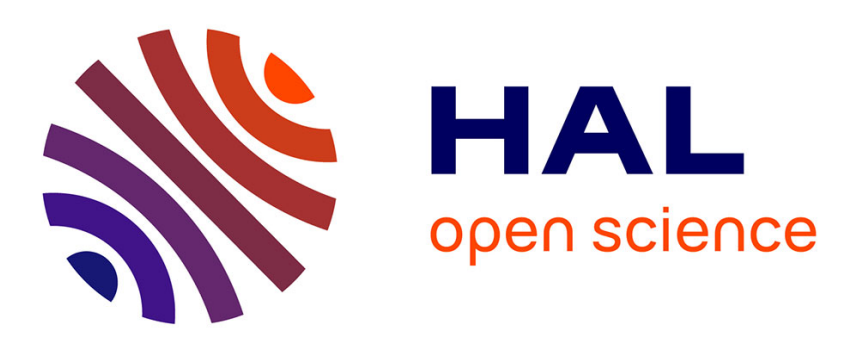

\title{
Optical response of a hematite coating: ellipsometry data versus Fourier-based computations
}

Enguerrand Couka, François Willot, Patrick Callet, Dominique Jeulin

\section{To cite this version:}

Enguerrand Couka, François Willot, Patrick Callet, Dominique Jeulin. Optical response of a hematite coating: ellipsometry data versus Fourier-based computations. Advanced Science, Engineering and Medicine, 2015, 7 (11), pp.925-931. 10.1166/asem.2015.1790 . hal-01232497

HAL Id: hal-01232497

https://hal-mines-paristech.archives-ouvertes.fr/hal-01232497

Submitted on 23 Nov 2015

HAL is a multi-disciplinary open access archive for the deposit and dissemination of scientific research documents, whether they are published or not. The documents may come from teaching and research institutions in France or abroad, or from public or private research centers.
L'archive ouverte pluridisciplinaire HAL, est destinée au dépôt et à la diffusion de documents scientifiques de niveau recherche, publiés ou non, émanant des établissements d'enseignement et de recherche français ou étrangers, des laboratoires publics ou privés. 


\title{
Optical response of a hematite coating: ellipsometry data vs. Fourier-based computations
}

\author{
E. Couka ${ }^{\mathrm{a} *}$, F. Willot ${ }^{\mathrm{a}}$, P. Callet ${ }^{\mathrm{b}, \mathrm{c}}$ and D. Jeulin ${ }^{\mathrm{a}}$ \\ ${ }^{a}$ Mines ParisTech, PSL Research University, Centre for Mathematical Morphology, 35 rue Saint-Honoré, \\ 77300 Fontainebleau, France, \\ ${ }^{b}$ Mines ParisTech, PSL Research University, Centre for Robotics, 60 Boulevard Saint-Michel, 75006 Paris, \\ France. \\ ${ }^{c}$ Applied Mathematics and Systems Laboratory, Centrale Supélec, 92295 Châtenay-Malabry, France.
}

$(20 / 01 / 2015)$

\begin{abstract}
The optical properties of a hematite-epoxy coating are predicted numerically and compared with ellipsometry measurements. The highly-heterogeneous dispersion of nanocubic hematite particles in the epoxy resin is simulated using a previously developed two-scales random model. The local anisotropic permittivity tensor of hematite particles, and that of the epoxy, are estimated by ellipsometry measurements carried out on a macroscopic hematite and epoxy samples. Fourier-based methods using a "discrete" Green operator are used to treat complex permittivities. They predict the effective and local electric displacement field in the quasi-static approximation. The former is close to two estimates based on the Hashin-Shtrikman bounds and a self-consistent approximation. Good agreement is found between experimental data and FFT computations in the whole range of the visible spectrum.
\end{abstract}

Keywords: Homogeneization; FFT method; Random microstructure models; Optical properties; Hematite coating; Ellipsometry

\section{Introduction}

Various forms of hematite crystals and polycristals have been synthesized and used in optical applications [3, 11, 26, 32, 33]. Synthesis techniques of hematite nanoparticles offer a wide range of dispersion [19] and particle shapes $[12,13]$. The spatial dispersion of particles, for which models have been proposed [7], is most often of a multiscale nature.

Both experimental and modeling techniques are useful to assess the effect of the shapes and spatial dispersion of the particles on the macroscopic properties. Energy loss techniques, carried out on thin films, allows one to estimate the complex permittivity of small regions in materials [24, 27]. The deconvolution of the signal is however challenging in the visible spectrum and optical properties remain difficult to measure at a small scale.

On the modeling side, numerical solutions of Mie's problem include Yee-cell techniques [6], which use finite differences in the time domain. In the quasi-static approximation of Maxwell's equations, effective medium theories such as Maxwell-Garnett's [18, 21], have been used to quantify the effect of nanoparticles on a material's optical properties [8, 28]. More involved homogenization procedures that rely on Helmholtz's equation have also been proposed [14, 15], or specific methods that apply to 3D periodic arrays [23]. Nevertheless, homogenization theories do not in general take into account the role of the multiscale distribution of nanoparticles in the material. In a recent numerical work, spectral approaches have been proposed in the quasi-static limit [1]. Although these methods allow

*Corresponding author. Email: enguerrand.couka@mines-paristech.fr 


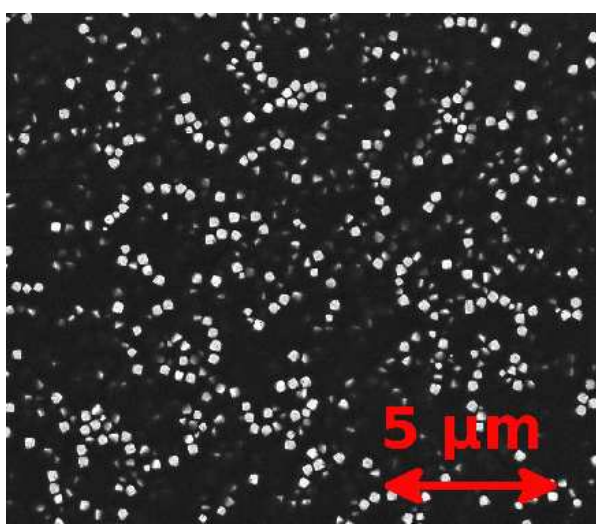

(a)

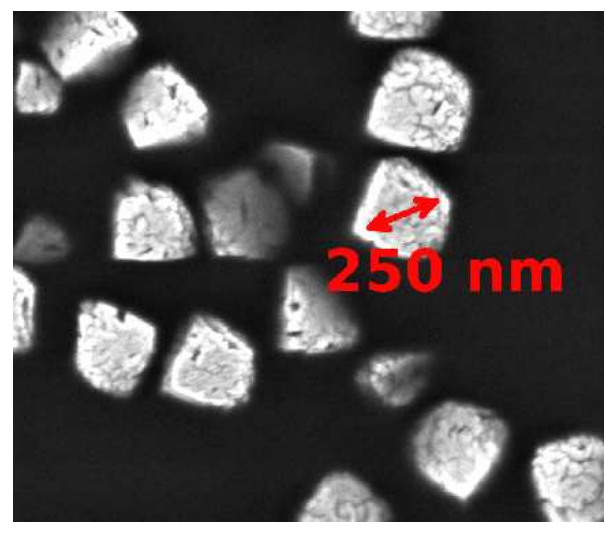

(b)

Figure 1. SEM images of the hematite-epoxy coating. White: hematite nanocubes; black: epoxy phase (image: M. Ben Achour, from [7]).

one to estimate the full-field properties of large random microstructures [29], they have not been confronted to optical measurements.

The object of interest of this work is a hematite-epoxy coating. The material contains pseudocubic hematite nanoparticles with a peculiar spatial dispersion (Fig. 1a) modeled numerically in a previous work [7]. As shown in scanning electron microscope images the particles typical size is about $250-300 \mathrm{~nm}$ (Fig. 1b). We refer to [25] for a detailed study of the structure of the cubic pigments. In the present work, a combined numerical and experimental approach is undertaken to study the optical properties of the hematite coating. It is organized as follows: ellipsometry measurements are described in Sec. (2) and FFT computations in Sec. (3). We compare measurements and modeling of the optical properties in Sec. (4). We conclude in Sec. (5).

\section{Ellipsometry measurements}

Spectroscopic ellipsometry measurement rely on the polarization of reflected light to compute the optical and the absorption indices. The general problem could be condensed in a short formulation: "inverting the Fresnel formulas". As the spectral reflectance of a sample of a smooth plane surface depends on the two spectral functions that are the real and the imaginary part of the complex index of refraction, we need an accurate measurement of these functions. This is possible using macroscopic samples as this optical characteristic is an intrinsic property. The general notion of index of refraction is only meaningful for homogeneous non-scattering materials. The measurement principle is briefly described hereafter.

A beam of light with known polarization is reflected on a smooth surface. The simultaneous measurement of the reflected intensity of a parallel beam of light and the change in polarization is recorded for a given angle of incidence and a given wavelength. As we have two spectral terms $(n, k)$ to be derived from these measurements we have to prepare the setup for accounting with the Fresnel conditions (perfectly smooth state of surface, sample planarity, parallel beam of light, accurate measurment of the angle of incidence, etc.). We refer to [10] for a general presentation of the spectroscopic ellipsometry technique.

All over the visible spectrum the magnetic permeability $\mu$ is always equal to unity so that we can write without any error in isotropic media, the relationship $n=\sqrt{\varepsilon \mu}$ linking the complex permittivity to the complex refactive index of the material, measured by spectroscopic ellipsometry. For anisotropic media, the second-order symmetric tensor $\varepsilon_{i j}(i, j=1 . .3)$ is linked to the refactive indices as follow:

$$
\left|n^{2} \delta_{i j}-n_{i} n_{j}-\varepsilon_{i j}\right|=0,
$$


where $|\cdot|$ represents the determinant [17].

The measurements related to hematite are carried out on a sample from the museum of mineralogy at Mines ParisTech (Fig. 2). Refractive indices are measured along two of its faces, orthogonal to each other, so as to take into account the crystal's anisotropy. The real and imaginary parts of the complex permittivity are represented in Fig. (3). By convention, we refer to the two observed directions as transversal and longitudinal. It was not possible to carry out the same measurement along a direction normal to the two considered here, without destroying the crystal. As shown in Fig. (3), the hematite cristal is anisotropic, nevertheless the difference between the two directions does not exceed $13 \%$ for the real part and $8 \%$ for the imaginary part. The average over both directions is shown as dotted lines.

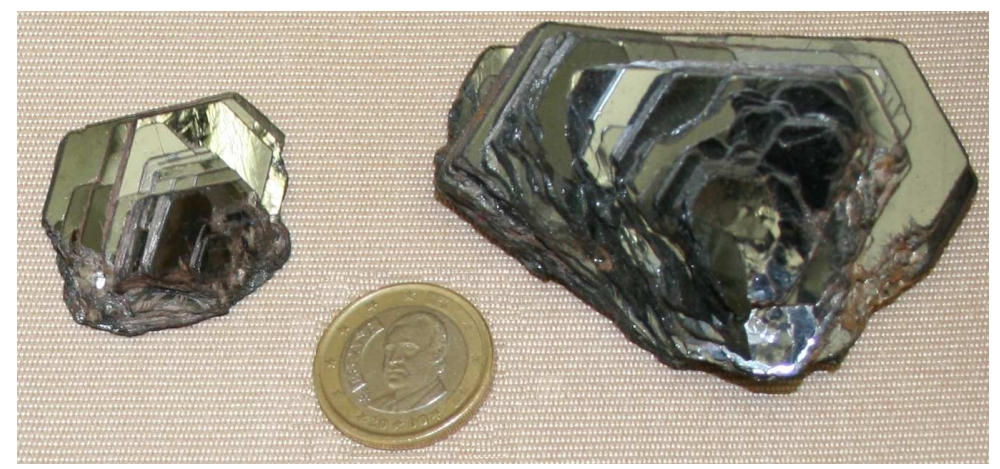

Figure 2. Rose-shaped hematite crystal with reflecting metallic gray aspect on the largest platelets and red appearance along the thickness. The latter is close to the deeply red streak of this material.

Refractive indices for the bulk epoxy phase are measured on a cylindrical sample of a few centimeters. Results are given in Fig. (4). The latter are an average over four measurements carried for different orientation of the sample. Very small variations are observed: the standard fluctuations are about $610^{-3} \mathrm{~S} / \mathrm{m}$ for all frequencies (not shown). The sample has been meticulously polished in order to minimize the influence of the surface roughness on the data. Nevertheless, characteristic oscillations of such surface effects are conspicuous for the imaginary part of the permittiviy, especially at small wavelengths, more sensitive to roughness. As expected, the real and imaginary parts of the complex permittivity of epoxy is much lower than that of hematite. The contrast of properties is about 100, for most frequencies, for the imaginary part. Indeed, epoxy is nearly transparent whereas the hematite crystal is metallic grey.

The permittivity of the hematite-epoxy coating is also measured using ellipsometry (Fig. 5). Error bars are provided by the standard fluctuations over four measurements, taken at different places on the sample. The measurements fall in-between that of its constituants. As expected, the real part of the complex permittivity of the coating is of the same order as that of epoxy, whereas the imaginary part is in-between that of the two phases. Furthermore, the characteristic points in the curves of the permittivity of hematite and of the coating match very well. For hematite, local maxima appear at $\lambda \approx 650 \mathrm{~nm}$ for the real part and at $\lambda \approx 480 \mathrm{~nm}$ for the imaginary part (Fig. 3). These maxima are observed at slightly different frequencies for the hematite coating (Fig. 5). A similar phenomenon holds for the slope changes of the permittivity of hematite, observed at $\lambda \approx 500$ $\mathrm{nm}$ for the real part and at $\lambda \approx 650 \mathrm{~nm}$ for the imaginary part (Fig. 3), also visible in Fig. (5).

\section{Fourier-based method in the quasi-static approximation}

We consider the following quasi-static approximation of the Maxwell equations:

$$
\nabla \cdot \mathbf{D}(\boldsymbol{x})=0, \quad \nabla \times \mathbf{E}(\boldsymbol{x})=0
$$




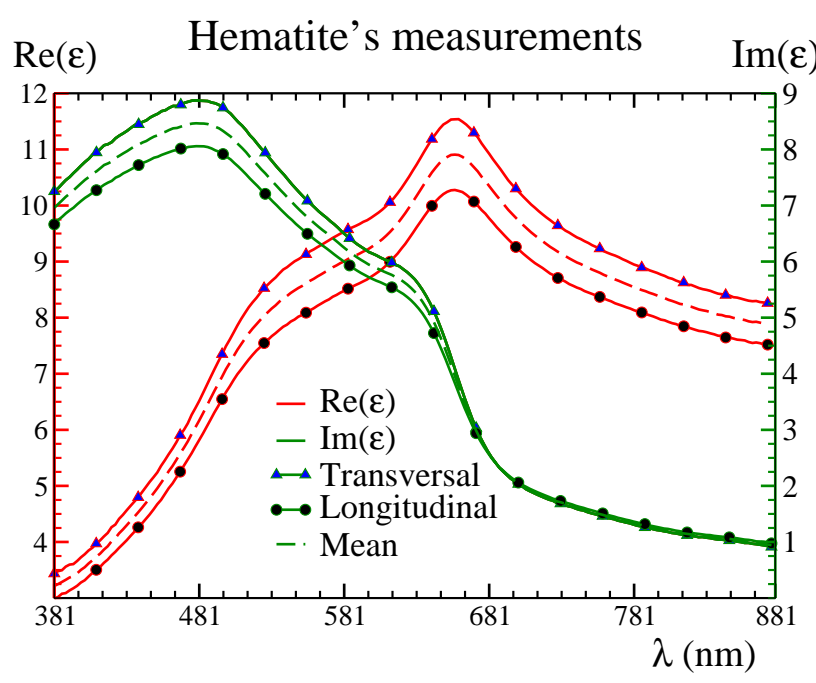

Figure 3. Ellipsometry measurements of the complex permittivity of a hematite crystal, along two orthogonal directions, in red (real part) and green (imaginary part), as a function of the light wavelength. Solid lines are guide to the eyes, the average over both direction is represented by dotted lines.

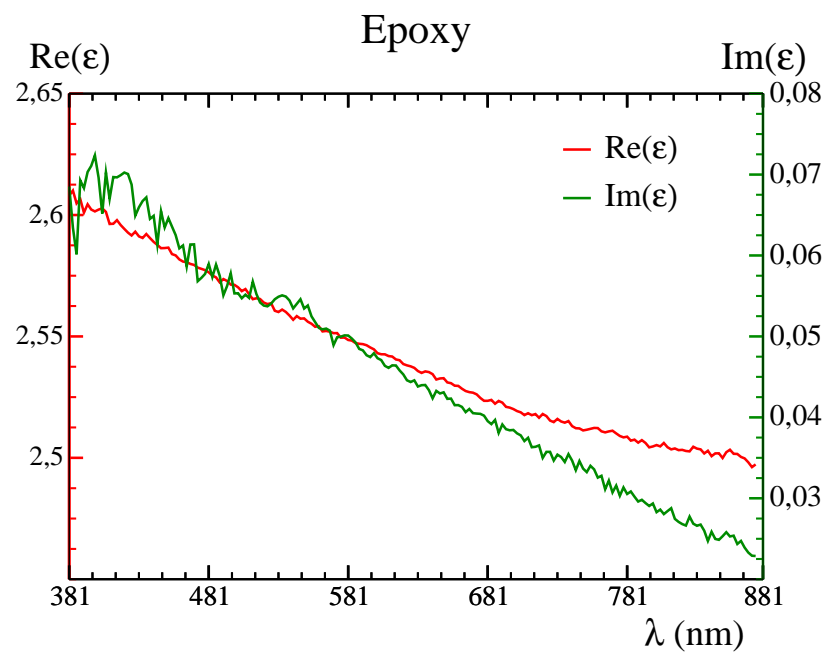

Figure 4. Ellipsometry measurements of the epoxy: real (red) and imaginary parts (green) of the complex permittivity.

where $\mathbf{D}(\boldsymbol{x})$ is the local electric displacement at point $\boldsymbol{x}, \mathbf{E}(\boldsymbol{x})$ is the local electric field, $\nabla \cdot$ is the divergence operator and $\nabla \times$ the curl operator. Eq. (1) is valid whenever the light wavelength $\lambda$ is much larger than the typical size $\xi$ of the heterogeneities in the material: $\lambda / \xi \gg 1$ [2]. In the two-scales microstructure considered in this work, hematite particles form necklaces around zones of diameters about $1.9 \mu \mathrm{m}$ [7]. Scale separation does not hold for $\xi=1.9 \mu \mathrm{m}$, However, the size of hematite particles $a=250 \mathrm{~nm}$ is significantly smaller than the wavelength, with $a / \lambda$ comprised between 0.3 and 0.6. Electrostatic is used in this work as a simple, low-cost approximation. In theory, electrodynamic effects apply in this domain for materials with isolated inclusions (see e.g. [4]). In any case, this approximation should be more accurate for large wavelengths (red) than for the smaller ones (blue, violet) and so we expect better results when $\lambda$ is large.

Neglecting polarization, the linear relation between electric and displacement fields is:

$$
D_{i}(\boldsymbol{x})=\varepsilon_{i j}(\boldsymbol{x}) E_{j}(\boldsymbol{x}),
$$

where $\varepsilon_{i j}(\boldsymbol{x})$ is the complex permittivity of the phase located at point $\boldsymbol{x}$. By convention, we denote by "phase 1" the hematite particles and by "phase 2" the embedding epoxy medium. The local 


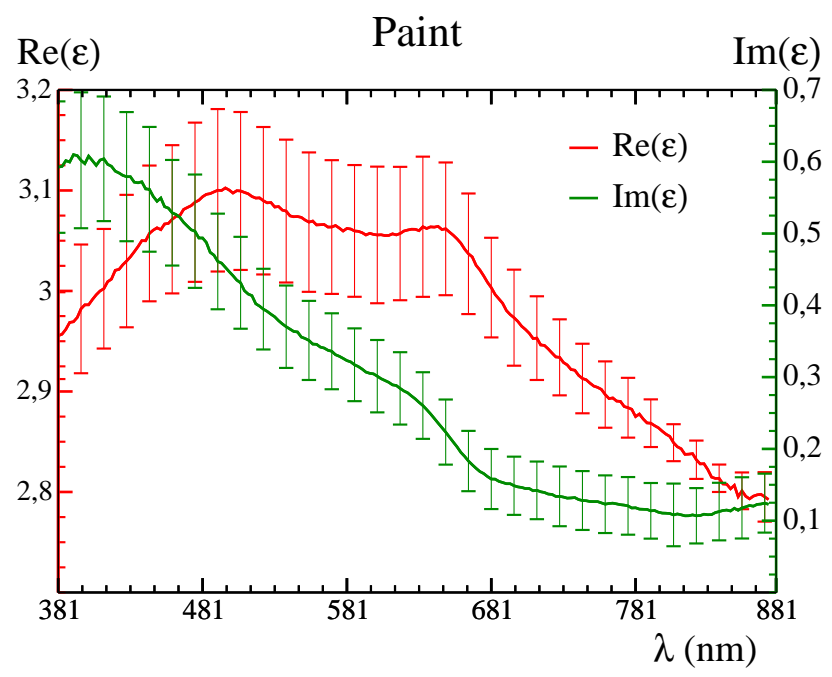

Figure 5. Real (red) and imaginary parts (green) of the complex permittivity of the hematite coating, measured by ellipsometry, as a function of the wavelength of light.

permittivity therefore reads:

$$
\varepsilon_{i j}(\boldsymbol{x})=\left\{\begin{array}{lc}
\varepsilon_{i j}^{(1)} & \text { if } \boldsymbol{x} \text { lies in phase 1 } \\
\varepsilon_{i j}^{(2)} & \text { otherwise }
\end{array}\right.
$$

In hematite particles, the permittivity tensor $\varepsilon^{(1)}$ is anisotropic whereas, in the epoxy, $\varepsilon^{(2)}$ is isotropic:

$$
\varepsilon_{i j}^{(2)}=\frac{\operatorname{tr}\left(\varepsilon^{(2)}\right)}{3} \delta_{i j}
$$

Eqs. (1) and (2) are solved in a cubic domain $\Omega$ of size $L$ with periodic boundary conditions:

$$
\mathbf{D}(\boldsymbol{x}) \cdot \boldsymbol{n}-\#, \quad \mathbf{E}(\boldsymbol{x}) \#, \quad \boldsymbol{x} \in \partial \Omega,
$$

where $\boldsymbol{n}$ is the local normal on the boundary $\partial \Omega$ of the domain, pointing outward, \# denotes periodicity and -\# anti-periodicity. The size $L$ is chosen so that $L \gg \xi$ and $\Omega$ is a representative volume element. Furthermore, the material is subjected to an applied (i.e. macroscopic) electric field $\overline{\mathbf{E}}$ :

$$
\langle\mathbf{E}\rangle_{\Omega}=\overline{\mathbf{E}}
$$

where $\langle\cdot\rangle_{\Omega}$ is the mean over $\Omega$. Using linearity, the mean electric displacement reads:

$$
\langle\mathbf{D}\rangle_{\Omega}=\widetilde{\varepsilon} \cdot \overline{\mathbf{E}},
$$

which defines the apparent complex permittivity $\widetilde{\varepsilon}$ of the volume element $\Omega$ [14]. The latter is also an estimate of the effective permittivity of the hematite coating.

The Fourier "accelerated scheme" [9] is used to solve Eqs. (1), (2) and (4). The latter consists in the following iterations, at step $k$ :

$$
\mathbf{E}^{k+1}=\mathbf{E}^{k}+2\left(\varepsilon+\varepsilon^{(0)}\right)^{-1} \cdot \varepsilon^{(0)} \cdot\left[\overline{\mathbf{E}}-\mathbf{E}^{k}-\mathbb{G}^{(0)} *\left(\varepsilon \cdot \mathbf{E}^{k}-\varepsilon^{(0)} \cdot \mathbf{E}^{k}\right)\right],
$$


where $\varepsilon^{(0)}$ is an arbitrary "reference" permittivity, $\mathbb{G}^{(0)}$ the associated Green operator and * denotes a convolution product, computed in the Fourier domain. At each iteration we treat the Fourier mode $\mathbf{q}=0$ separately and enforce $\mathbf{E}(q=0)=\overline{\mathbf{E}}$. For simplicity, we assume that $\boldsymbol{\varepsilon}_{i j}^{(0)}=\varepsilon^{(0)} \delta_{i j}$ is isotropic and identify it with a scalar. We set $\varepsilon^{(0)}=-0.6$ for the reference permittivity, at all frequencies. We use the modified Green operator from [31], introduced in the context of conductivity:

$$
G_{i j}^{(0)}(\boldsymbol{k})=\frac{k_{i} k_{j}^{*}}{\varepsilon^{(0)} k_{i} k_{i}^{*}}, \quad k_{i}=\mathrm{i}\left(1-\mathrm{e}^{\mathrm{i} q_{i}}\right),
$$

where $q_{i}=0,2 \pi / L, \ldots, 2 \pi(L-1) / L$ is the Fourier wave vector and * denotes the complex conjugate and $\mathbf{i}=\sqrt{-1}$. Use of $\mathbf{k}$ above amounts to discretize (1) by finite differences. Improved convergence properties as well as more accurate local fields have been observed with this method [31]. In optics, the FFT scheme (7) is similar to that in conductivity, except that the electric and displacement fields $\mathbf{E}$ and $\mathbf{D}$, and the permittivity $\varepsilon$, are complex quantities [1]. Discrete Fourier transforms are used to switch between the real space and Fourier domain.

For both methods, iterations are stopped when the following convergence criterion is met:

$$
\frac{\sqrt{\left\langle|\boldsymbol{k}(\boldsymbol{q}) \cdot \mathbf{D}(\boldsymbol{q})|^{2}\right\rangle_{\boldsymbol{q}}}}{\mathbf{D}(\boldsymbol{q}=0)}<\eta
$$

where $\langle\cdot\rangle_{\boldsymbol{q}}$ is a mean over $\boldsymbol{q}$ in the Fourier domain, $\mathbf{D}(\boldsymbol{q}=0)=\langle\mathbf{D}(\boldsymbol{x})\rangle_{\Omega}$ is the mean electric displacement and $\eta=10^{-10}$ is the required precision. The above enforces conservation of the displacement field.

In the numerical computations carried out hereafter, we first assume that the crystal orientations of each nanocube are distributed uniformly on the sphere and uncorrelated:

$$
\varepsilon_{i j}^{(1)}=R_{i k} R_{j l} \varepsilon_{k l}^{(\mathrm{r})}
$$

where the tensors $\boldsymbol{R}$ are 3D rotation matrix that are unique to each nanoparticle and the components of $\varepsilon^{(\mathrm{r})}$ are to be determined from measurements. Making use of the two previously obtained "transverse" and "longitudinal" components, we assume the following transversely-isotropic form for $\varepsilon^{(\mathrm{r})}$ :

$$
\varepsilon_{k l}^{(\mathrm{r})}=\left(\begin{array}{ccc}
\varepsilon_{\|} & 0 & 0 \\
0 & \varepsilon_{\perp} & 0 \\
0 & 0 & \varepsilon_{\perp}
\end{array}\right)
$$

where $\varepsilon_{\|}$and $\varepsilon_{\perp}$ depend on the frequency, and are given in Fig. (3).

For $\lambda=661 \mathrm{~nm}$, using $\varepsilon_{\|}=8.51+1.61 \mathrm{i}, \varepsilon_{\perp}=9.40+1.56 \mathrm{i}$ and FFT computations on a domain $\Omega$ discretized on a grid of $128^{3}$ voxels, the macroscopic permittivity tensor $\widetilde{\varepsilon}^{\prime}$ reads, in matrix form:

$$
\begin{aligned}
\widetilde{\varepsilon} & =\left(\begin{array}{ccc}
2.92 & -1.8610^{-2} & -1.6410^{-2} \\
-1.8610^{-2} & 2.91 & -1.4010^{-2} \\
-1.6410^{-2} & -1.4010^{-2} & 2.92
\end{array}\right)+\left(\begin{array}{ccc}
9.7 & -0.47 & -0.42 \\
-0.47 & 9.6 & -0.32 \\
-0.42 & -0.31 & 9.7
\end{array}\right) 10^{-2} \mathrm{i} \\
& \approx(2.92+0.097 \mathrm{i}) \delta_{i j} .
\end{aligned}
$$

The effective response computed above is close to an isotropic law, because the crystal orientations are uniform on the sphere. We compare the above results with a simplified isotropic law where we 
approximate:

$$
\varepsilon_{k l}^{(\mathrm{r})} \approx \varepsilon^{(\mathrm{r})} \delta_{k l}=\frac{1}{2}\left(\varepsilon_{\|}+\varepsilon_{\perp}\right)
$$

Taking again $\lambda=661 \mathrm{~nm}$, we have now $\varepsilon_{k l}^{(\mathrm{r})}=8.96+1.58 \mathrm{i}$ and FFT results provide $\widetilde{\varepsilon}=2.91+0.098 \mathrm{i}$, close to the effective permittivity computed in the anisotropic case (12). Accordingly, we use the approximation (13) in all computations carried out hereafter.

For comparisons, we also use the following estimates:

$$
\varepsilon_{\mathrm{HS}}=\varepsilon^{(2)}+\frac{3 p \varepsilon^{(2)}\left(\varepsilon^{(1)}-\varepsilon^{(2)}\right)}{3 \varepsilon^{(2)}+(1-p)\left(\varepsilon^{(1)}-\varepsilon^{(2)}\right)}, \quad p \frac{\varepsilon^{(1)}-\varepsilon_{\mathrm{SC}}}{\varepsilon^{(1)}+2 \varepsilon_{\mathrm{SC}}}+(1-p) \frac{\varepsilon^{(2)}-\varepsilon_{\mathrm{SC}}}{\varepsilon^{(2)}+2 \varepsilon_{\mathrm{SC}}}=0,
$$

where $p \approx 10 \%$ is the volume fraction of hematite in the coating. In Eq. (14) above, $\varepsilon_{\mathrm{HS}}$ is the exact result for the Hashin coating assemblage where phase 1 (the particles) is embedded into phase 2 (the epoxy). This estimate is located on the frontier of the complex domain that delimits the Hashin and Shtrikman bounds [22]. Furthermore, $\varepsilon_{\mathrm{SC}}$ refers to the self-consistent approximation, based on the requirement that the perturbations of the fields due to the inclusions vanish, on average [5].

\section{FFT results vs. ellipsometry data}

In the following, a sample of the 3D random model containing 99886 nanoparticles is discretized on a grid of $500^{3}$ voxels at resolution $31 \mathrm{~nm} /$ voxel, so that each nanoparticles is discretized as a cube of about $5^{3}$ voxels. A $2 \mathrm{D}$ section of the material is represented in Fig. (6). The visible spectrum is discretized along 24 equispaced values for the wavelength $\lambda=381, . ., 781 \mathrm{~nm}$. FFT field maps of the electric displacement component $D_{1}$ are represented in Fig. (7) for the three frequencies $\lambda=401$, 600 , and $801 \mathrm{~nm}$. We apply a macroscopic electric field with components $\bar{E}_{1}=1, \bar{E}_{2}=\bar{E}_{3}=0$ so that the field $D_{1}$ is "parallel" to the applied field. The 1-axis is oriented top to bottom on the maps. To highlight the fields's patterns, $0.1 \%$ of the highest values of the dielectric field are thresholded. The lowest values are shown in blue, and the highest in red, with green and yellow in-between. Additionally, each map includes a 2D section (top-right quadrant) of a microstructure with high resolution $31 \mathrm{~nm} /$ voxel, discretized on a grid of $512^{3}$ voxels. As expected, the real and imaginary parts of the dielectric component $D_{1}$ are higher in hematite particles than in the epoxy phase. As shown in the high-resolution maps, the dielectric field inside the nanoparticles is quite heterogenous. In some of the particles, the dielectric field is more important along a band joining two corners or opposite faces, as already observed in previous studies $([1,30])$. The lowest values appear in dark blue in the epoxy close. The latter are in regions close to particles located outside of the $2 \mathrm{D}$ section.

In order to give an interval of confidence of the effective permittivity estimated by FFT, we used the approach based on the RVE (representative volume element [16]), for the three wavelengths $\lambda=441,621,801 \mathrm{~nm}$. The latter is based on the asymptotic expansion [20]:

$$
\frac{D_{\varepsilon}^{2}(V)}{D_{\varepsilon}^{2}} \sim \frac{A_{3}}{V}
$$

where $D_{\boldsymbol{\varepsilon}}^{2}(V)$ is the variance of the apparent permittivity $\varepsilon$ estimated on a volume $V, D_{\varepsilon}^{2}$ is the point variance and $A_{3}$ is the integral range. The relative error on the estimate for the effective permittivity $\widetilde{\varepsilon}$ reads:

$$
e_{r e l}=\sqrt{\frac{4 D_{\varepsilon}^{2} A_{3}}{V \widetilde{\varepsilon}(\lambda)}}
$$




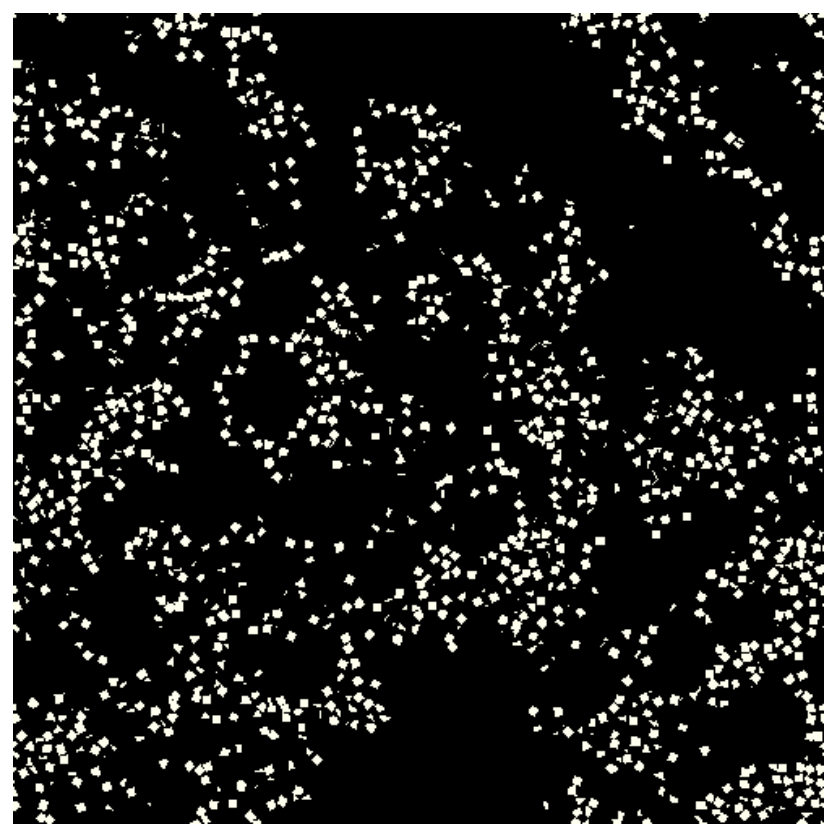

Figure 6. 2D section of the 3D microstructure model showing hematite (white) embedded in the epoxy matrix (black).

The variance $D_{\varepsilon}^{2}(V)$ is estimated by averaging on the FFT fields over subvolumes of varying sizes. The expansion (15) is recovered when the subvolume $V$ contains more than $200^{3}$ voxels and the integral range is determined from numerical fits on FFT data. For the real and imaginary parts of the permittivity:

$$
\begin{aligned}
& A_{3}^{\operatorname{Re}}= \begin{cases}25^{3} \text { voxels }\left(0.775^{3} \mu \mathrm{m}^{3}\right) & \text { if } \lambda=441 \mathrm{~nm}, \\
24^{3} \text { voxels }\left(0.744^{3} \mu \mathrm{m}^{3}\right) & \text { if } \lambda=621 \mathrm{~nm}, \\
22^{3} \text { voxels }\left(0.682^{3} \mu \mathrm{m}^{3}\right) & \text { if } \lambda=800 \mathrm{~nm},\end{cases} \\
& A_{3}^{\operatorname{Im}}=\left\{\begin{array}{cl}
21^{3} \text { voxels }\left(0.651^{3} \mu \mathrm{m}^{3}\right) & \text { if } \lambda=441 \mathrm{~nm}, \\
7^{3} \text { voxels }\left(0.217^{3} \mu \mathrm{m}^{3}\right) & \text { if } \lambda=621 \mathrm{~nm}, \\
6^{3} \text { voxels }\left(0.186^{3} \mu \mathrm{m}^{3}\right) & \text { if } \lambda=800 \mathrm{~nm},
\end{array}\right.
\end{aligned}
$$

so that the relative errors on the FFT estimate are:

$$
e_{\mathrm{rel}}^{\mathrm{Re}}=\left\{\begin{array}{ll}
1.29 \% & \text { if } \lambda=441 \mathrm{~nm}, \\
1.27 \% & \text { if } \lambda=621 \mathrm{~nm}, \\
0.92 \% & \text { if } \lambda=800 \mathrm{~nm}
\end{array} \quad e_{\mathrm{rel}}^{\mathrm{Im}}=\left\{\begin{array}{cc}
2.51 \% & \text { if } \lambda=441 \mathrm{~nm}, \\
0.21 \% & \text { if } \lambda=621 \mathrm{~nm}, \\
0.36 \% & \text { if } \lambda=800 \mathrm{~nm}
\end{array}\right.\right.
$$

Conversely, when $\lambda=441 \mathrm{~nm}$, a volume size $V=4285^{3}$ voxels or $132.8 \mu \mathrm{m}^{3}$ is necessary to achieve a precision of $0.1 \%$ for the imaginary part of the effective permittivity. The largest RVEs are observed for the lowest wavelengths (ex: $\lambda=441 \mathrm{~nm}$ ).

FFT results for the effective permittivity $\widetilde{\varepsilon}$ are represented in Figs. (8) and (9) for the real and imaginary parts, respectively, and compared to ellipsometry data. Error bars for the FFT results, computed as above, are shown in black. FFT results are close to the Hashin and Shtrikman and self-consistent analytical estimates, shown in green and orange in the figures. This validates our use of the quasistatic approximation, as made for the estimates. For the real part, the FFT results are also slightly lower than measurements for the coating, however the difference between the two is not more than $3.1 \%$, of the same order as the standard deviations for the ellipsometry measurements.

Similar observations hold for the imaginary part of the complex permittivity (Fig. 9). FFT results are close to the Hashin and Shtrikman estimates. Also, the relative difference between FFT 


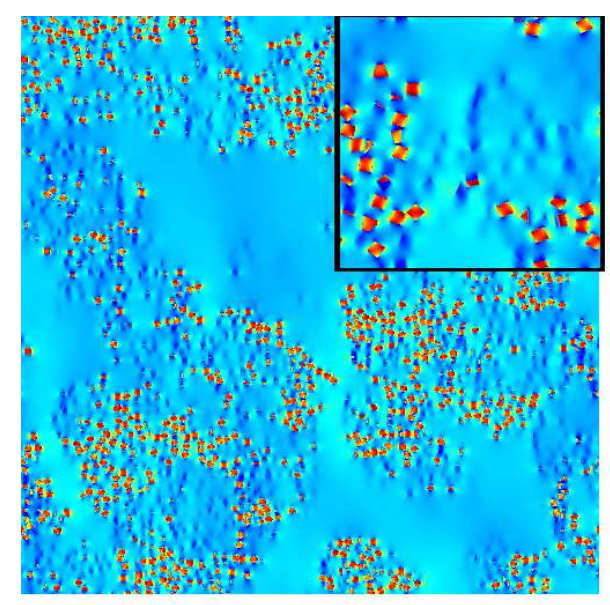

(a) $\operatorname{Re}\left(D_{1}\right), \lambda=401 \mathrm{~nm}$

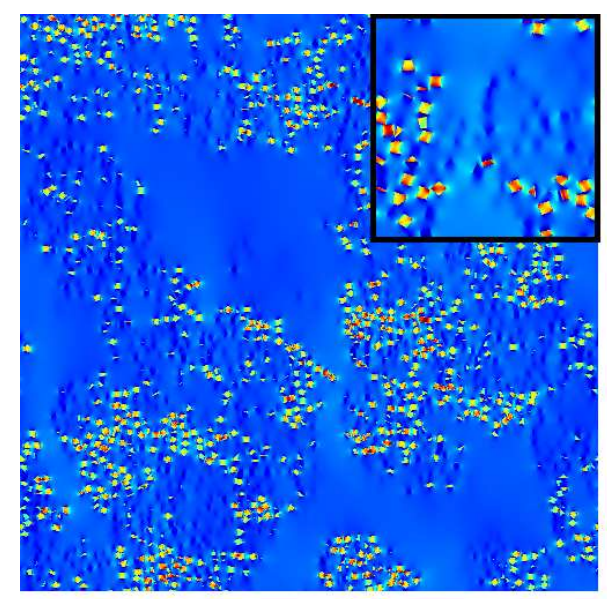

(c) $\operatorname{Re}\left(D_{1}\right), \lambda=600 \mathrm{~nm}$

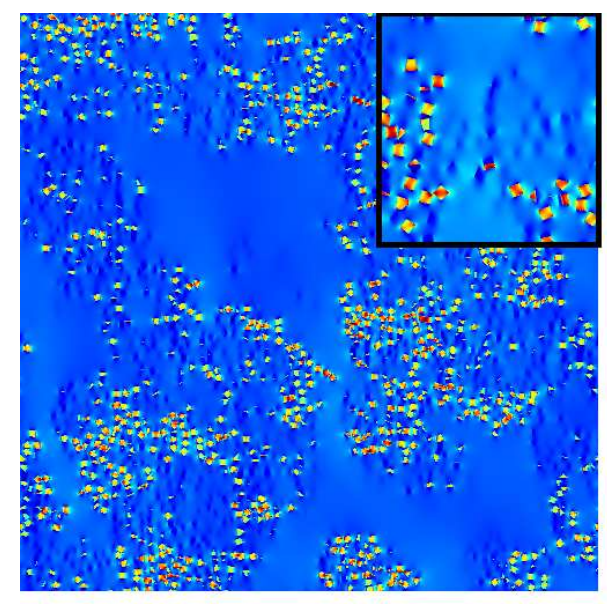

(e) $\operatorname{Re}\left(D_{1}\right), \lambda=801 \mathrm{~nm}$

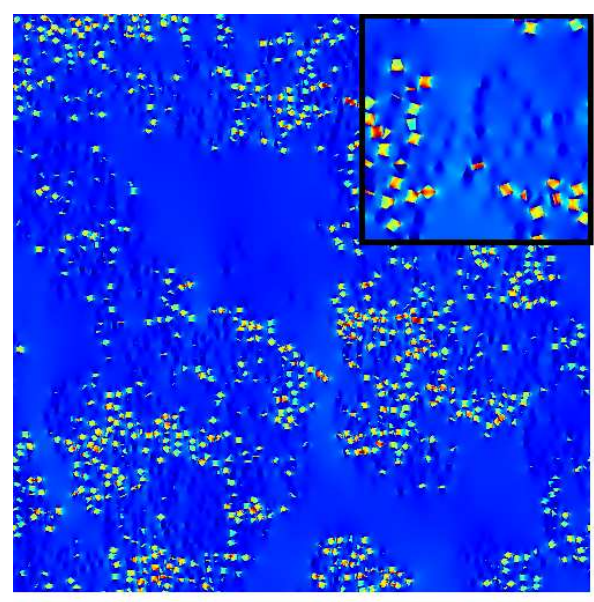

(b) $\operatorname{Im}\left(D_{1}\right), \lambda=401 \mathrm{~nm}$

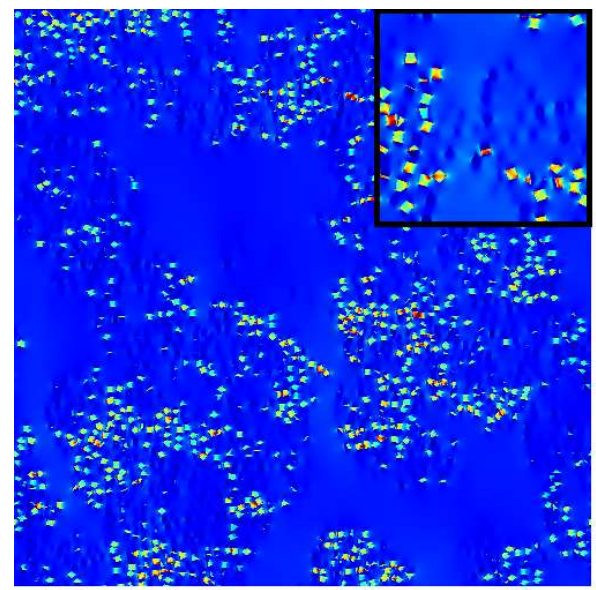

(d) $\operatorname{Im}\left(D_{1}\right), \lambda=600 \mathrm{~nm}$

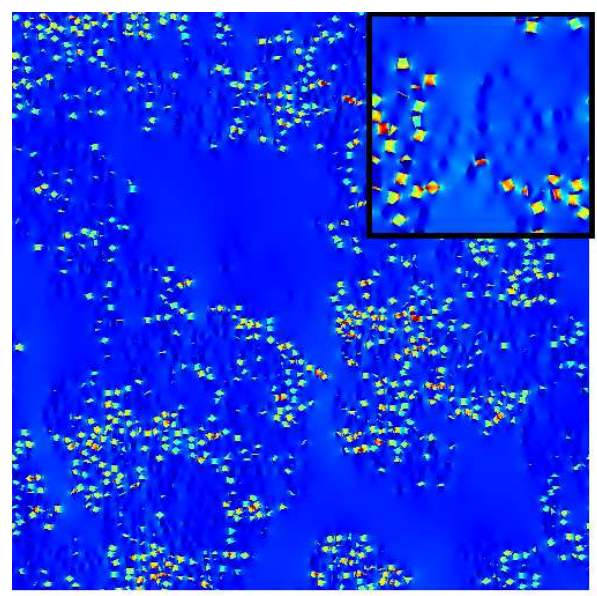

(f) $\operatorname{Im}\left(D_{1}\right), \lambda=801 \mathrm{~nm}$

Figure 7. 2D sections of the real and imaginary parts of the component $D_{1}$ of the electric displacement field, parallel to the applied loading $\bar{E}_{1}=1$ at three frequencies $\lambda=401,600$ and $801 \mathrm{~nm}$. Microstructure resolution: $31 \mathrm{~nm} /$ voxel discretized on a grid of $500^{3}$ voxels. Inset: higher resolution $6.2 \mathrm{~nm} /$ voxel, discretized on a grid of $512^{3}$ voxels. 
data and ellipsometry is about $18 \%$ for small wavelengths, and increases up to $40 \%$ at the highest wavelengths. Nevertheless, FFT results are within the error estimates of the ellipsometry data.

In the numerical FFT computations, ellipsometry measurements carried out on a hematite crystal have been used for the local complex permittivity of the nanoparticles. However, we emphasize that the two complex permittivities are nresumahly different

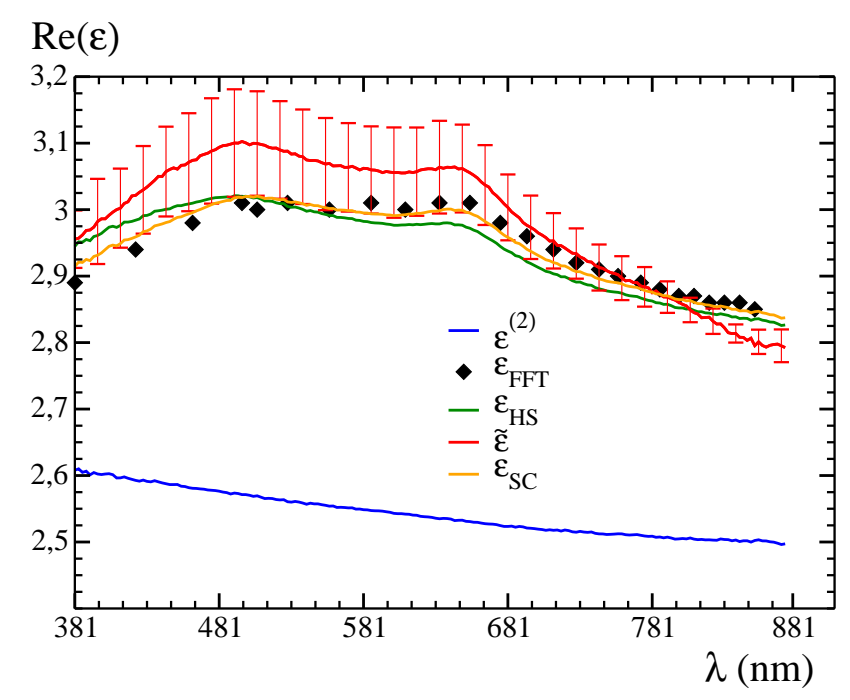

Figure 8. Real part of the complex permittivity $\widetilde{\varepsilon}$ of the hematite coating: Hashin and Shtrikman estimate (green), self consistent estimate (orange), ellipsometry measurements (red) and numerical FFT predictions (black symbols). Blue, for comparison: ellipsometry measurements of the real part of the complex permittivity of the epoxy.

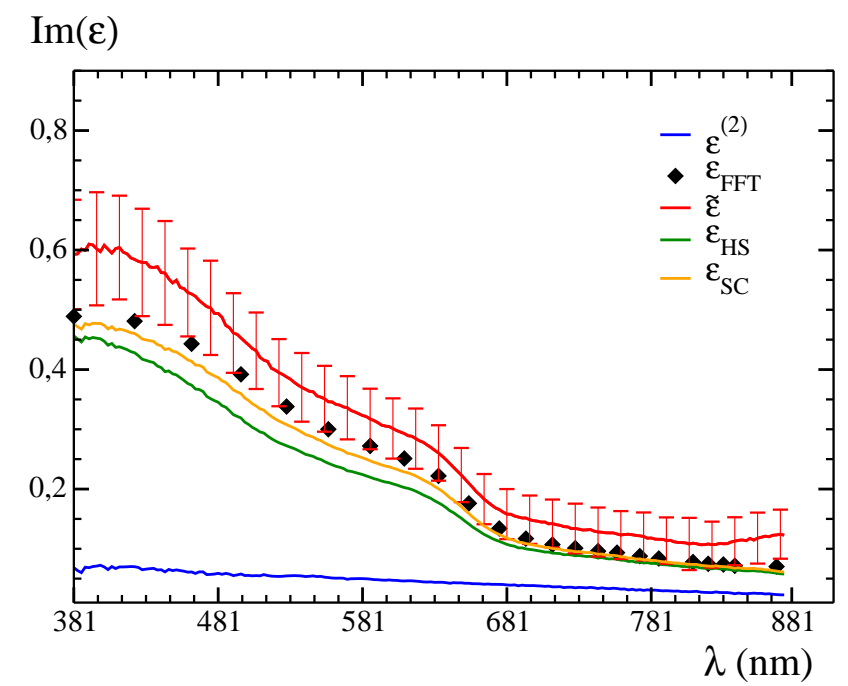

Figure 9. Continuation of Fig. (8): imaginary part of the complex permittivity $\widetilde{\varepsilon}$ of the hematite coating.

\section{Conclusion}

This work focuses on a hematite coating made of hematite nanoparticles embedded in a epoxy phase, with $10 \%$ volume fraction for the hematite particles. We measured the complex permittivity of the coating, epoxy phase, and hematite crystal using ellipsometry. Experimental results indicate an anisotropic complex permittivity for the hematite crystal. The latter two are used in 
numerical Fourier-based computations to predict the optical properties of the coating. In these FFT calculations, we used the quasi-static approximation of the Maxwell equations. To take into account the multiscale dispersion of hematite in the coating, a 3D model morphological model previously developed was used in the FFT computations. Although anisotropic properties were observed for the hematite crystal, numerical computations indicate that the anisotropy has little effects on the macroscopic properties of the coating. Overall, the FFT predictions are found to be close to the ellipsometry measurements. The highest differences are observed for the imaginary part of the complex permittivity at the lowest wavelength. This result is consistent with the quasistatic assumption, valid at low wavelengths. However, the highest relative differences are observed at high wavelengths, suggesting that a difference exists between the optical response of hematite nanoparticles and that of the macroscopic crystal. Nevertheless, the present work shows that FFT computations are an efficient tool for predicting the optical properties of nanocomposites.

\section{Acknowledgement}

This study was made with the support of A.N.R. (Agence Nationale de la Recherche) under grant 20284 (LIMA project). The authors are grateful to M. Ben Achour, A. Chesnaud, J.-F. Hochepied and A. Thorel for providing images of the hematite coating, surface polishing, synthesis and preparation of the samples.

\section{References}

[1] Azzimonti, D. F.;Willot, F.;Jeulin, D. Optical properties of deposit models for paints: full-fields FFT computations and representative volume element. Journal of Modern Optics. 2013, 60(7), 1-10.

[2] Berthier, S. In Optique des Milieux Composites, Ed. Polytechnica, Paris, 1993.

[3] Bhuiyan, T.I.; Nakanishi, M.; Kusano, Y.; Fujii, T.; Takada, J.; Ikeda, Y. Synthesis, morphology and color tone properties of the lanthanum substituted hematite. Materials Letters. 2007, 61(17), 3774-3777.

[4] Bohren, C.; Huffman, D. In Absorption and Scattering of Light by Small Particles, Ed. Wiley, New-York, 1983.

[5] Bruggeman, V. D. Berechnung verschiedener physikalischer Konstanten von heterogenen Substanzen. I. Dielektrizitätskonstanten und Leitfähigkeiten der Mischkörper aus isotropen Substanzen. Annalen der Physik. 1935, 416(7), 636-664.

[6] Chen, Y.; Mittra, R.; Harms, P. Finite-difference time-domain algorithm for solving Maxwell's equations in rotationally symmetric geometries. Microwave Theory and Techniques. IEEE Transactions. 1996, 44(6), 832-839.

[7] Couka, E.; Willot, F.; Jeulin, D.; Ben Achour, M.; Chesnaud, A.; Thorel, A. Modeling of the multiscale dispersion of nanoparticles in a hematite coating. Journal of Nanoscience and Nanotechnology 2015, 15(5), 3515-3521.

[8] Cummings, K.D.; Garland, J.C.; Tanner, D.B. Optical properties of a small-particle composite. Physical Review B. 1984, 30(8), 4170.

[9] Eyre, D.J.; Milton, G.W. A fast numerical scheme for computing the response of composites using grid refinement. The European Physical Journal - Applied Physics. 1999, 6(1), 41-47.

[10] Fujiwara, H. Spectroscopic ellipsometry. Principles and applications. Wiley. Tokyo, 2007.

[11] Glasscock, J.A.; Barnes, P.R.F.; Plumb, I.C.; Bendavid, A.; Martin, P.J. Structural, optical and electrical properties of undoped polycrystalline hematite thin films produced using filtered arc deposition. Thin Solid Films. 2008, 516(8), 1716-1724.

[12] Han, Y.; Wang, Y.; Li, L.; Wang, Y.; Jiao, L.; Yuan, H.; Liu, S. Preparation and electrochemical performance of flower-like hematite for lithium-ion batteries. Electrochimica Acta. 2011, 56(9), 31753181.

[13] Huang, X.; Guan, J.; Xiao, Z.; Tong, G.; Mou, F.; Fan, X.A. Flower-like porous hematite nanoarchitectures achieved by complexation-mediated oxidation-hydrolysis reaction. Journal of colloid and interface science. 2011, 357(1), 36-45. 
[14] Kanaun, S.K.; Jeulin, D. Effective field method in the problem of electromagnetic wave propagation through the medium with spherical inclusions. Journal of Electromagnetic Waves and Applications. 1997, 11(11), 1531-1566.

[15] Kanaun, S.K. Dielectric properties properties of matrix composite materials with high volume concentration of inclusions (effective field approach). International Journal of Engineering Science. 2003, 41, $1287-1312$.

[16] Kanit, T.; Forest, S.; Galliet, I.; Mounoury, V.; Jeulin, D. Determination of the size of the representative volume element for random composites: statistical and numerical approach. International Journal of Solids and Structures. 2003, 13-14(40), 3647-3679.

[17] Landau, L.D.; Lipschtiz, E.M. Course of Theoretical Physics: Vol. 8 : Electrodynamics of Continuous Media, chapter XI. Permagon Press., Oxford, 1960.

[18] Levy, O.; Stroud, D. Maxwell Garnett theory for mixtures of anisotropic inclusions: Application to conducting polymers. Physical Review B. 1997, 56(13), 8035.

[19] Manickavasagam, S.; Saltiel, C.; Giesche, H. Characterization of colloidal hematite particle shape and dispersion behavior. Journal of Colloid and Interface Science. 2004, 280(2), 417-430.

[20] Matheron, G. The theory of regionalized variables and its applications. Paris School of Mines Publications 1971, Paris.

[21] Maxwell-Garnett, J.C. Effective medium models for the optical properties of inhomogeneous media. Philosophical Transactions of the Royal Society of London. 1904, B 203, pp. 385.

[22] Milton, G.W. Bounds on the complex permittivity of a two-component composite material Journal of Applied Physics 1981, 52(8), 5286-5293.

[23] Mochán, W.L.; Ortiz, G.P.; Mendoza, B.S. Efficient homogenization procedure for the calculation of optical properties of 3D nanostructures composites. Optics express. 2010, 18(21), 22119-22127.

[24] Paciornik, S.; Gomes, O.d.F.M.; Delarue, A.; Schamm, S.; Jeulin, D.; Thorel, A. Multi-scale analysis of the dielectric properties and structure of resin/carbon-black nanocomposites. The European Physical Journal Applied Physics. 2003, 21(01), 17-26.

[25] Park, G.; Shindo, D.; Waseda, Y.; Sugimoto, T. Internal Structure Analysis of Monodispersed Pseudocubic Hematite Particles by Electron Microscopy. Journal of colloid and interface science. 1996, 177(1), 198-207.

[26] Spuch-Calvar, M.; Pérez-Juste, J.; Liz-Marzán, L.M. Hematite spindles with optical functionalities: Growth of gold nanoshells and assembly of gold nanorods. Journal of Colloid and Interface Science. 2007, 310, 297-301.

[27] Suenaga, K.; Bouchet, D.; Colliex, C.; Thorel, A.; Brandon, D.G. Investigations of alumina/spinel and alumina/zirconia interfaces by spatially resolved electron energy loss spectroscopy. Journal of the European Ceramic Society. 1998, 18(10), 1453-1459.

[28] Tuncer, E.; Serdyuk, Y.V.; Gubanski, S.M. Dielectric mixtures: electrical properties and modeling. Dielectrics and Electrical Insulation. IEEE Transactions. 2002, 9(5), 809-828.

[29] Willot, F.; Jeulin, D. Elastic and electrical behavior of some random multiscale highly-contrasted composites. Journal of Multiscale Computational Engineering. 2010, 9(3), 305-326.

[30] Willot, F.; Gillibert, L.; Jeulin, D. Microstructure-induced hotspots in the thermal and elastic responses of granular media. International Journal of Solids and Structures. 2013, 50(10), 1699-1709.

[31] Willot, F.; Abdallah, B; Pellegrini, Y-P. Fourier-based schemes with modified Green operator for computing the electrical response of heterogeneous media with accurate local fields. International Journal for Numerical Methods in Engineering. 2014, 98(7), 518-533.

[32] Xu, Y.; Yang, S.; Zhang, G.; Sun, Y.; Gao, D.; Sun, Y. Uniform hematite $\alpha-\mathrm{Fe}_{2} \mathrm{O}_{3}$ nanoparticles: Morphology, size-controlled hydrothermal synthesis and formation mechanism. Materials Letters. 2011, 65, 1911-1914.

[33] Zhang, G.Y.; Feng, Y.; Xu, Y.Y.; Gao, D.Z.; Sun, Y.Q. Controlled synthesis of mesoporous $\alpha-\mathrm{Fe}_{2} \mathrm{O}_{3}$ nanorods and visible light photocatalytic property. Materials Research Bulletin 2012, 47(3), 625-630. 